\title{
Reentrant Klystron Cavities with Circular and Conical Inserts
}

\author{
Joaquim J. Barroso and Joaquim P. Leite Neto \\ Associated Plasma Laboratory, National Institute for Space Research - INPE \\ 12201-970 São José dos Campos, SP, Brazil
}

\section{Introduction}

Having a simple mechanical construction and wide tuning range, narrow gap reentrant cylindrical cavities were originally investigated during the development years of the klystron [1] and have since then been widely used for the construction of microwave oscillators, and tunable resonators. These cavities allow the realization of resonant frequencies in the low $\mathrm{GHz}$ range without the need for large physical dimensions, with the ridged gap having the effect of reducing the frequency and focusing the electric field (Fig.1).

A reentrant cavity, as viewed in Fig. 1, is basically an LC resonator where the equivalent capacitance is determined by the gap spacing between the coaxial post (radius $r_{1}$ ) and the top closing plate (radius $r_{2}$ ), with the inductance being mainly due to coaxial region $\left(r_{2}-r_{1}\right)$ of the cavity. Since the exact solutions of Maxwell's equations inside the resonator configuration are not known, the properties of interest (resonance frequency, and quality $Q$ factor) must be calculated numerically. By using the code Superfish [3], the present paper examines how the resonance frequency $\mathrm{f}_{0}$ and the electrical Q factor relate to the geometrical shape of the coaxial insert.
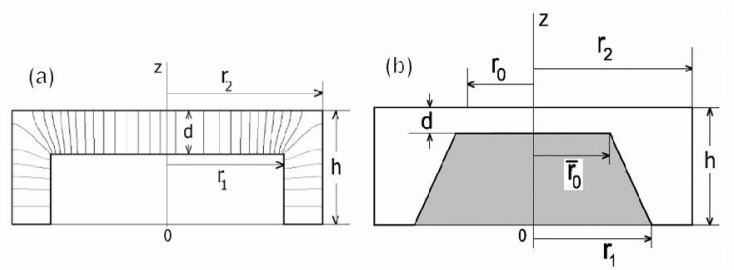

Figure 1. Reentrant cavities with (a) circular cylindrical and (b) conical inserts

\section{Resonance frequency and Ohmic $Q$ factor}

At an electrical conductivity of $5.810^{8} \mathrm{~S} / \mathrm{m}$, we compare in Fig.2 the resonance frequency and Ohmic Q factor arising from three inserts of the same height placed in a cylindrical cavity $8.0 \mathrm{~cm}$ in diameter. The associated tuning curves (Fig. 2) relating resonance frequency to axial gap are nearly superimposed up to $\mathrm{d}=0.50 \mathrm{~mm}$, and so the thin cylinder turns out to be equivalent to the conical insert regarding tuning characteristics. This equivalence, however, does not hold as far as loss effects are concerned (Fig. 3). For $\mathrm{d}>0.50 \mathrm{~mm}$ the $Q$ factors corresponding to the conical post are systematically 30 percent higher than those associated with the thin cylinder.

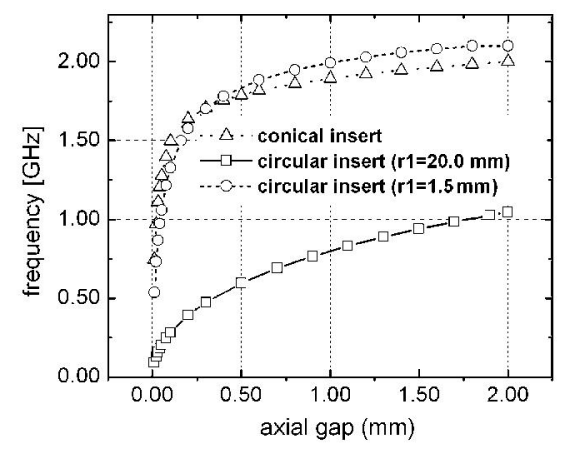

Figure 2. Resonance frequencies as function of the axial gap spacing in reentrant cavities

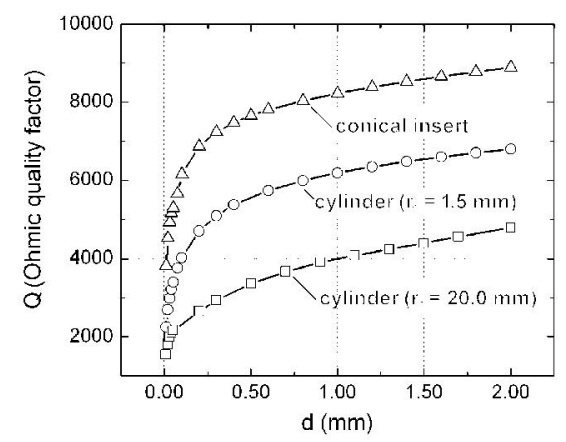

Figure 3. Ohmic Q factors $\left(\sigma=5.810^{8} \mathrm{~S} / \mathrm{m}\right)$ of reentrant cavities as function of the axial gap spacing

At a fixed gap spacing $\mathrm{d}=2.0 \mathrm{~mm}$, the effect of the inner radius $r_{1}$ on $\mathrm{f}_{0}$ is displayed in Figs. 4 and 5 . First in Fig. 5, letting $r_{1}=0$ or $r_{1}=r_{2}=40.0 \mathrm{~mm}$ readily recovers for the resulting $\mathrm{TM}_{010}$-mode circular cavity the resonance frequency of 2.868 $\mathrm{GHz}$, given by $\left.(15 / \pi) x_{01} / r_{2}(\mathrm{~cm})\right]$, where $x_{01}=2.4048$ is the first zero of the Bessel function $\mathrm{J}_{0}(\mathrm{x})$. In between the limits $0<r_{1}<r_{2}$ what happens with the frequency $f_{0}\left(r_{1}\right)$ ? This question can be answered 
on the basis of Slater's theorem [2], which states that when a cavity is perturbed on its boundaries (as by pushing a small part of the wall in or out) the original resonance frequency $f_{0}$ changes to a perturbed value $f_{l}$ according to

$$
f_{1}=f_{0} \sqrt{1+\frac{\Delta W_{m}-\Delta W_{e}}{W_{m}+W_{e}}}
$$

where $\Delta W_{m}$ and $\Delta W_{e}$ are the magnetic and electrical energies in the perturbing volume. Let us take two representative points on the $f_{0}\left(r_{1}\right)$ curve in Fig. 4. At $r_{1}=10.0 \mathrm{~mm}$, a small perturbation of $\Delta r_{1}=+0.5$ $\mathrm{mm}$ is made by pushing in the cylindrical surface toward the cavity's outer wall. Then we verify which amount of energy, either electrical or magnetic, is larger inside the perturbing volume. A calculation from Superfish [3] gives a negative value for the term $\Delta W_{m}-\Delta W_{e}$ in (1), thus indicating that $f_{0}$ decreases as $r_{1}$ is increased. On the other hand, at $r_{I}=36.0 \mathrm{~mm}$ the term $\Delta W_{m}-\Delta W_{e}$ turns out to be positive which explains why the right-hand side of the $f_{0}\left(r_{1}\right)$ curve increases monotonically with $r_{1}$. At the lowest frequency $(\triangleright 1.03 \mathrm{GHz})$ the energy difference vanishes in the neighboring of the corresponding cylindrical surface of radius $r_{1}$ $\triangleright 22.5 \mathrm{~mm}$. Then we see in Fig. 4 that over the range $0 £ r_{1} \downarrow r_{2}$ the frequency tuning is as large as $\Delta f \triangleright 1.8 \mathrm{GHz}$. This is in sharp contrast with the cavity of conical insert, for which the corresponding frequency tuning is constrained to a narrow band of $\Delta f \triangleright 0.2 \mathrm{GHz}$, as displayed in Fig. 5 . Nevertheless, the cavity of conical insert outperforms the cavity with circular cylinder regarding Ohmic losses. As illustrated in Fig. 6, the coaxially-tapered cavity provides $Q$ factors typically 50 percent higher. In Fig. 6, Superfish results for the circular cavity are compared with an analytical formula described in [2].

\section{References}

[1] W. Hansen, J. Appl. Phys., 10: 38-45, 1939.

[2] J. J. Barroso, P. J. Castro, J. P. L. Neto, and O. D. Aguiar, Int. J. Infrared Millimeter Waves, 26(8):1071-1083, August 2005.

[3] J. H. Billen and L. Young, Poisson/Superfish", Rep. LA-UR-96-1934, LANL, Los Alamos, NM, 1996.

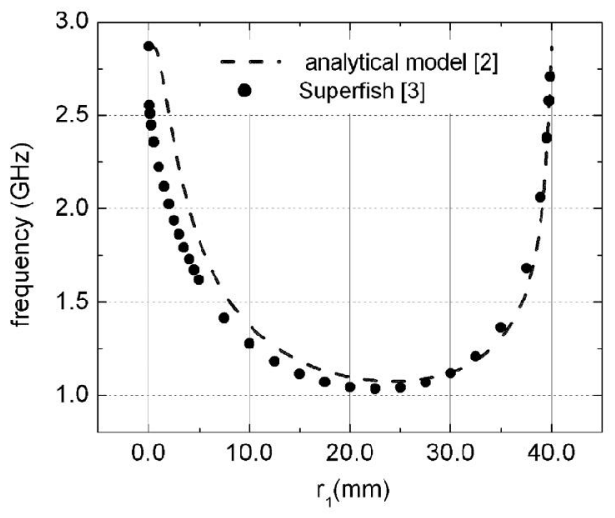

Figure 4. Resonance frequency dependence on the inner radius $r_{1}$ for a circular cavity with $h=20.0 \mathrm{~mm}$, $r_{2}=40.0 \mathrm{~mm}$, and $d=2.0 \mathrm{~mm}$.

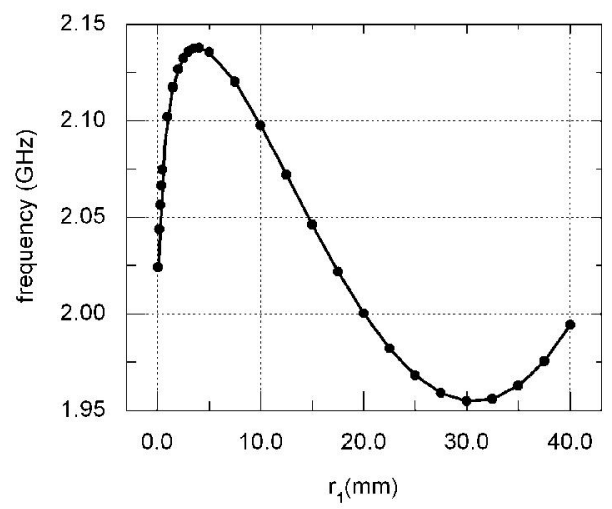

Figure 5. Resonance frequency dependence on the inner radius $r_{1}$ for the cavity with conical insert ( $h=20.0 \mathrm{~mm}, r_{2}=40.0 \mathrm{~mm}$, and $d=2.0 \mathrm{~mm}$ ).

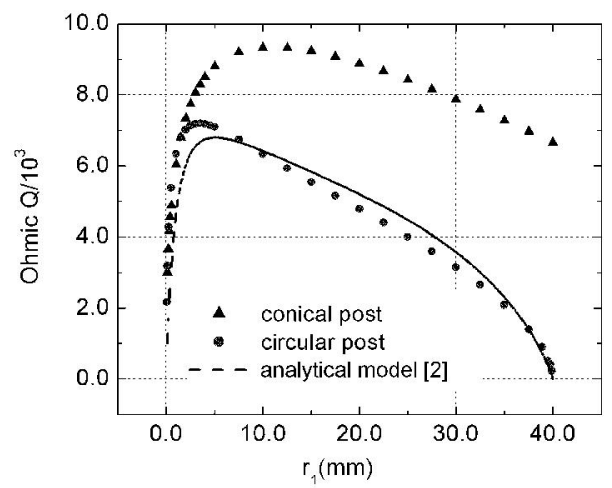

Figure 6. As function of the inner radius $r_{1}$, the Ohmic Q factor for the cavities with conical and circular inserts. 